\title{
In vivo three-dimensional photoacoustic tomography of a whole mouse head
}

\author{
Kwang Hyun Song \\ Optical Imaging Laboratory, Department of Biomedical Engineering, Texas A\&M University, \\ College Station, Texas 77843 \\ George Stoica \\ Department of Pathobiology, Texas A\&M University, College Station, Texas 77843 \\ Lihong V. Wang \\ Optical Imaging Laboratory, Department of Biomedical Engineering, Texas A\&M University, \\ College Station, Texas 77843
}

Received April 4, 2006; revised May 15, 2006; accepted May 17, 2006; posted May 26, 2006 (Doc. ID 69679); published July 25, 2006

\begin{abstract}
An in vivo photoacoustic imaging system was designed and implemented to image the entire small animal head. A special scanning gantry was designed to enable in vivo imaging in coronal cross sections with high contrast and good spatial resolution for the first time to our knowledge. By use of a $2.25 \mathrm{MHz}$ ultrasonic transducer with a $6 \mathrm{~mm}$ diameter active element, an in-plane radial resolution of $\sim 312 \mu \mathrm{m}$ was achieved. Deeply seated arterial and venous vessels in the head measuring up to $1.7 \mathrm{~cm}$ in diameter were simultaneously imaged in vivo with $804 \mathrm{~nm}$ wavelength laser excitation of photoacoustic waves. @ 2006 Optical Society of America
\end{abstract}

OCIS codes: $170.5120,170.3880,170.0110$.

Photoacoustic tomography (PAT) is a novel imaging modality that is nonionizing and highly sensitive to optical absorption of biological tissue, especially blood in vessels. ${ }^{1}$ In addition, PAT has a deeper penetration depth than other high-resolution pure optical imaging modalities, ${ }^{2,3}$ because the latter lose high-resolution imaging information beyond approximately one transport mean free path as a result of strong light scattering, whereas the former remains unaffected. PAT combines the merits of both optical and ultrasonic imaging and hence provides both high optical contrast and good ultrasonic resolution. ${ }^{4-7}$

PAT has been successfully applied to visualize the structures of a number of different biological tissues. Using PAT, Wang et al. obtained in vivo images of cerebral blood vessels on the cortex of a rat brain where the vessels were within $2-3 \mathrm{~mm}$ of the tissue surface. ${ }^{6,7}$ They presented in situ three-dimensional mouse brain imaging by using the same system, but the resolution for deep structures was far inferior to that for shallow vessels. ${ }^{8}$ Kruger et al. demonstrated in situ whole-head three-dimensional imaging of a nude mouse by use of an ultrasonic transducer array. ${ }^{9}$ In their study the deeply located blood vessels, such as the circle of Willis, anterior cerebral artery, and other arterial vessels, were not imaged with good spatial resolution or high contrast, even though the superficial venous vessels, such as the jugular vein around the head, were clearly imaged. In the present study we designed an in vivo whole-mousehead PAT system. We successfully imaged in vivo deep arterial vessels as well as superficial venous vessels with high contrast and good spatial resolution in coronal views.
Figure 1 shows a schematic of the newly designed system. To maximize the optical penetration depth and to select an isosbestic point of molar extinction coefficient of oxy- and deoxy-hemoglobin, we chose an $804 \mathrm{~nm}$ wavelength output as the excitation source from a $Q$-switched Nd:YAG laser (LS-2137/2, LOTIS TII)-pumped tunable Ti:sapphire laser (LT-2211A, LOTIS TII). To illuminate efficiently, the excitation light was divided by a beam splitter (BS1-784-50, CVI Laser) into two beams, which were then deliv-

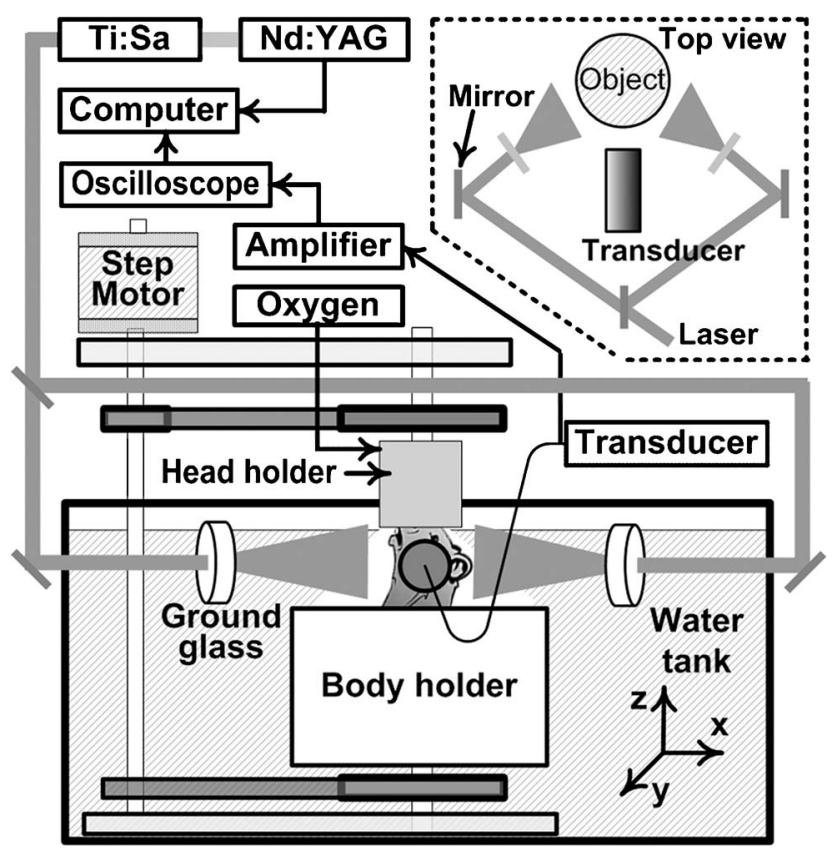

Fig. 1. Schematic of the experimental setup of photoacoustic tomography for in vivo whole-head small animal imaging. Sa, sapphire. 
ered to the sample by two mirrors and homogenized by ground glasses. The pulsed laser energy was maintained below the maximum permissible exposure for skin at the $804 \mathrm{~nm}$ wavelength ${ }^{10}$ ( $\sim 15.5 \mathrm{~mJ} / \mathrm{cm}^{2}$ for each beam). To avoid generation of strong photoacoustic (PA) signals from the surface of the object near the ultrasonic transducer, we applied side illumination in a spirit similar to dark-field illumination. ${ }^{11}$

A high signal-to-noise ratio is indispensable for detecting weak PA signals from locations deep within an object. Therefore we used a $2.25 \mathrm{MHz}$ ultrasonic transducer (V323, Panametrics, Waltham, Mass.) with a $6 \mathrm{~mm}$ diameter active element and a $65.6 \%$ bandwidth at $6 \mathrm{~dB}$, where the relatively low frequency and large active area are conducive to a high signal-to-noise ratio. A flat transducer was employed in place of a focused one to allow the application of the modified back projection algorithm for image reconstruction. ${ }^{12}$ To minimize the interference of surface signals and maximize the detection of deep signals from the head, we placed the ultrasonic transducer between two beams. Finally, the computer acquired all of the amplified and digitized signals and reconstructed the distribution of the optical absorption in the imaging plane.

Two particular devices in the scanning gantry, the rotation stages and mouse holders, as shown in Fig. 1 , were designed in house for in vivo imaging. The rotation stages have two parts, one located at the top and the other at the bottom, which rotate at the same angular speed. The mouse holders are composed of a head holder, which fixes the teeth and provides oxygen, and a body holder, which fixes the body and maintains the body temperature. The body holder is waterproof because the animal and the ultrasonic transducer are immersed in water to couple the PA waves from the animal to the ultrasonic transducer. The scanning radius of the ultrasonic transducer is $4 \mathrm{~cm}$.

To evaluate the image resolution of the designed system, we imaged nine carbon fibers ( $6 \mu \mathrm{m}$ in diameter) and two human hairs (30 $\mu \mathrm{m}$ in diameter) fixed in a $15 \%$ porcine gelatin with $0.5 \%$ Intralipid- $20 \%$, the reduced scattering coefficient of which was estimated to be $2.1 \mathrm{~cm}^{-1}$. ${ }^{13}$ Figure 2(a) shows the PAT image of the phantom; bright spots indicate the high optical absorption of the carbon fibers and hairs. Figure 2(b) shows the radial and tangential resolutions measured from the carbon fibers in the image. The radial resolution, defined by the FWHM of the main lobe, is approximately $312 \pm 5 \mu \mathrm{m}$ (standard error), which is close to the theoretically predicted value. ${ }^{12}$ The tangential resolution of the system deteriorates as the imaging point moves away from the imaging center owing to the aperture effect. ${ }^{5,12}$

A nude mouse (Harlan Company) weighing approximately $20 \mathrm{~g}$ was employed for in vivo head imaging. General anesthesia was subcutaneously administered to the nude mouse every hour. During data acquisition, pure oxygen was provided through the head holder. Figure 3 shows the threedimensional whole-head in vivo images of the nude
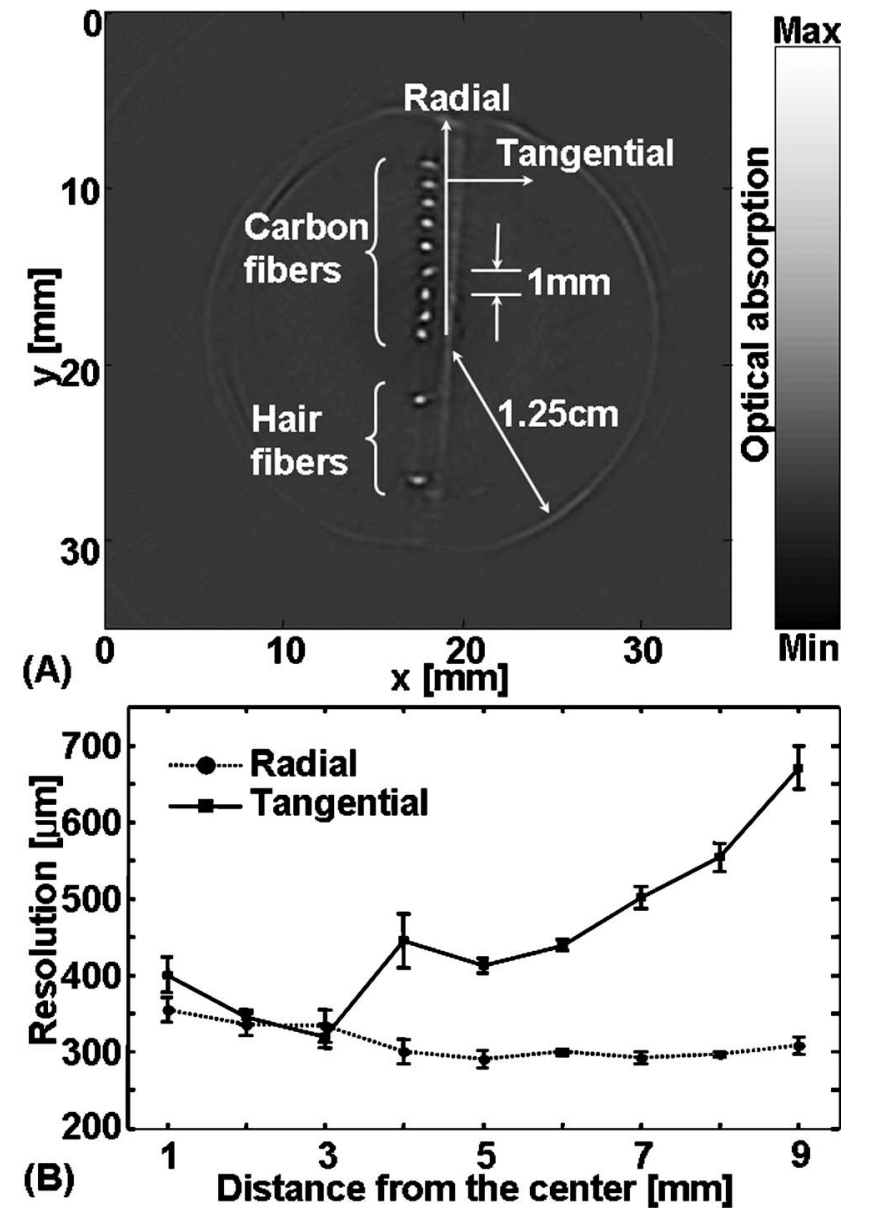

Fig. 2. (A) PAT image of nine carbon fibers and two hairs in gelatin. Objects are perpendicular to the imaging plane. (Note that fibers are not exactly perpendicular to the imaging plane, so distortion exists due to the slice thickness.) The grayscale bar indicates the magnitude of optical absorption. (B) Radial and tangential resolutions with standard errors. Dashed curve with circles, radial resolution; solid curve with squares, tangential resolution, showing the aperture effect of the transducer.

mouse in the coronal view. Because of the side illumination, light energy exponentially decays from the surface inward, which results in generation of much weaker signals in deep regions. To compensate for light attenuation in the images, we estimated the fluence distribution of light in the head by using a Monte Carlo simulation and then normalized the reconstructed PA image by using a two-dimensional fluence map. ${ }^{14}$ Because hemoglobin is the strongest optical absorber in the near-IR spectral region and generates strong PA signals, the most prominent structures in PAT images are blood vessels. Most of the blood vessels in a head are branches of the carotid artery and the jugular vein. Here the major blood vessels were segmented with heuristically chosen thresholds such that the segmented major vessels appear in all of the pseudocolor images, which are then superimposed on the original grayscale images. Some key vessels are identified as shown in Fig. 3.

For whole-head PAT to be used in practical applications, the time resolution and the slice thickness need to be improved. The current data acquisition 
time for one slice was $13 \mathrm{~min}$, which can be shortened with an ultrasonic transducer array system. The imaging system employed here had a large slice thickness for two reasons: the wide reception angle of the transducer in the elevation direction and the strong light scattering inside the biological tissue. The slice thickness (elevation resolution) is approximately equal to the diameter of the ultrasonic transducer. A cylindrically focused transducer or a threedimensional reconstruction algorithm can be used to
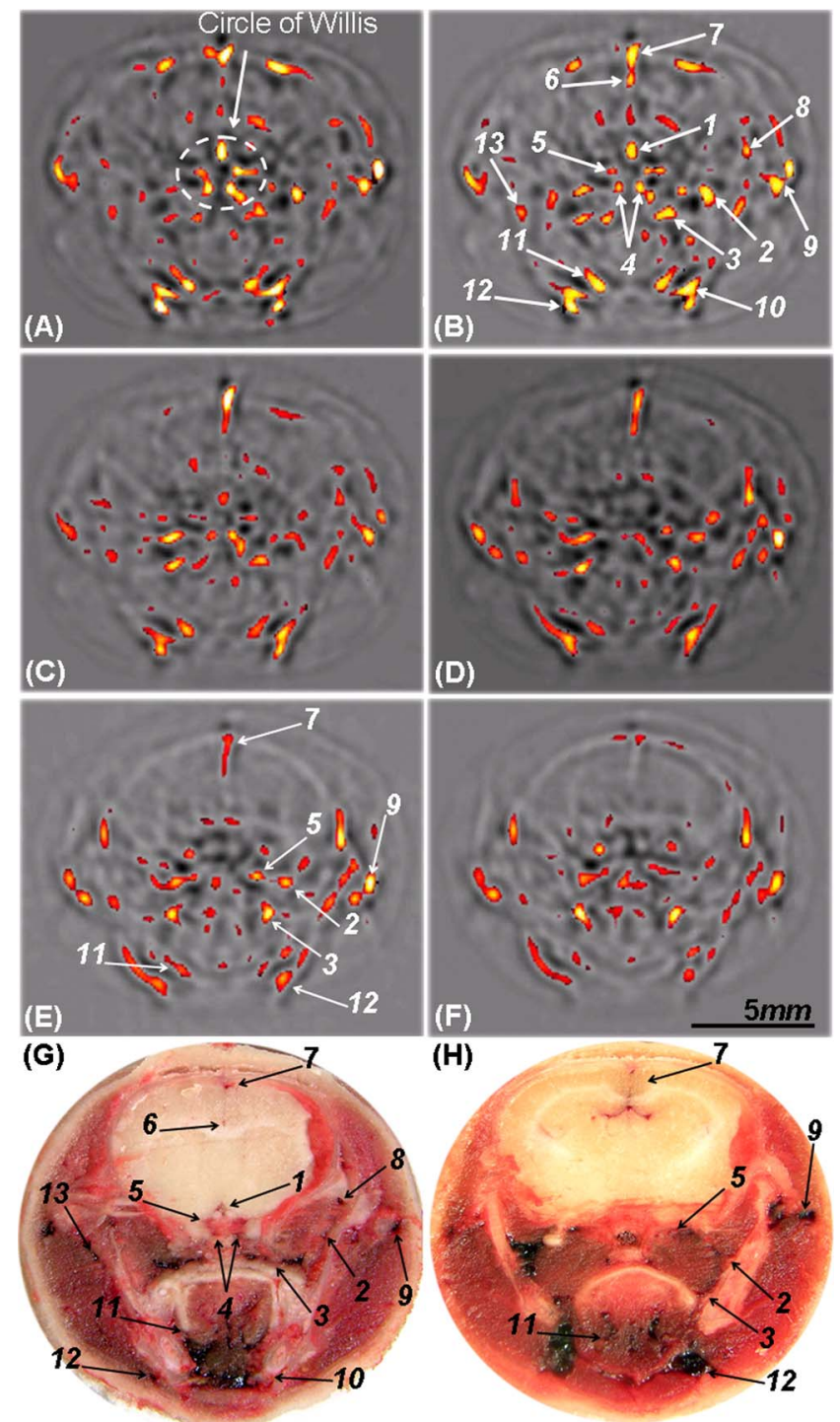

Fig. 3. (Color online) In vivo three-dimensional wholehead photoacoustic tomography of a nude mouse in coronal views. Images were obtained from the anterior to the posterior parts of the brain with a depth separation of $1 \mathrm{~mm}$ between adjacent scans. Arrows indicate higher optical absorption in blood vessels. The photographs $(\mathrm{G})$ and $(\mathrm{H})$ of the cross sections were taken after PAT data acquisition for comparison with corresponding PAT images (B) and (E), respectively. 1, Anterior cerebral artery; 2, mandibular alveolar artery; 3, 4, arterial vessels; 5 , posterior communication artery; 6, internal cerebral artery; 7, median fissure; 8, venous vessel; 9, facial vein; 10, facial artery; 11, sublingual artery; 12, jugular vein; 13, venous vessel. minimize the slice thickness. The reconstruction algorithm adopted here assumed that the distribution of light fluence and acoustic speed were homogeneous in the imaging plane. However, because of the side illumination, the distribution of light fluence was, in fact, inhomogeneous. In addition, when PA signals travel through heterogeneous biological tissues, such as brain, muscle, blood vessels, skin, and trachea, they suffer from acoustic speed aberrations. To achieve higher accuracy, the reconstruction algorithm needs to be modified to consider these two types of heterogeneity.

In this study we accomplished in vivo threedimensional whole-head imaging by using PAT. We were able to image deep arterial vessels, such as the circle of Willis, as well as superficial venous vessels with good spatial resolution and high contrast in coronal views in vivo for the first time to our knowledge. Previous studies have been confined to superficial or in situ imaging. We believe that our work can be extended to the study of cerebral hemodynamic changes by simultaneously measuring oxygen saturation and cerebral blood volume, the two key parameters for monitoring brain activities related to brain tumors, ischemia, stroke, etc. ${ }^{15}$

We thank Jung-Taek Oh, Meng-Lin Li, Konstantin Maslov, Geng Ku, and Xueyi Xie for technical assistance and Sergiu V. Similache for assistance with animal handling. The project was sponsored by $\mathrm{Na}-$ tional Institutes of Health grants R01 EB000712 and R01 NS46214. L. Wang's email address is lwang @tamu.edu; K. H. Song's is khsong@tamu.edu.

\section{References}

1. S. L. Jacques and S. A. Prahl, "Absorption spectra for biological tissues," http://omlc.ogi.edu/.

2. V. Ntziachristos, J. Ripoll, L.-H. Wang, and R. Weissleder, Nat. Biotechnol. 23, 313 (2005).

3. G. Ku and L.-H. Wang, Opt. Lett. 30, 507 (2005).

4. R. O. Esenaliev, A. A. Karabutov, and A. A. Oraevsky, IEEE J. Sel. Top. Quantum Electron. 5, 981 (1999).

5. G. Ku, X. Wang, G. Stoica, and L.-H. Wang, Phys. Med. Biol. 49, 1329 (2004).

6. X. Wang, Y. Pang, G. Ku, X. Xie, G. Stoica, and L.-H. Wang, Nat. Biotechnol. 21, 803 (2003).

7. X. Wang, G. Ku, M. A. Wegiel, D. J. Bornhop, G. Stoica, and L.-H. Wang, Opt. Lett. 29, 730 (2004).

8. X. Wang and L.-H. Wang, Opt. Lett. 28, 1739 (2003).

9. R. A. Kruger, W. L. Kiser Jr., D. R. Reinecke, G. A. Kruger, and K. D. Miller, Mol. Imaging 2, 113 (2003).

10. American National Standards Institute, "American national standard for the safe use of lasers," ANSI Z136.1 (American National Standards Institute, 2000).

11. K. Maslov, G. Stoica, and L.-H. Wang, Opt. Lett. 30, 625 (2005)

12. M. Xu and L.-H. Wang, Phys. Rev. E 67, 056605 (2003).

13. S. T. Flock, S. L. Jacques, B. C. Wilson, W. M. Star, and M. J. C. van Gemert, Lasers Surg. Med. 12, 510 (1992).

14. L.-H. Wang, S. L. Jacques, and L. Zheng, Comput. Methods Programs Biomed. 47, 131 (1995).

15. J. F. Adam, H. Elleaume, G. L. Duc, S. Corde, A. M. Charvet, I. Troprès, J. F. L. Bas, and F. Estève, J. Cereb. Blood Flow Metab. 23, 499 (2003). 